\title{
EXPERIENCE WITH DEUTERIUM-TRITIUM PLASMAS HEATED BY HIGH POWER NEUTRAL BEAMS
}

\author{
L. R. Grisham, J. H. Kamperschroer, T. O'Connor, M. Oldaker, \\ T. Stevenson, A. Von Halle, and the TFTR Group \\ Princeton University Plasma Physics Laboratory \\ P. O. Box 451, Princeton, N. J. 08543, U. S. A.
}

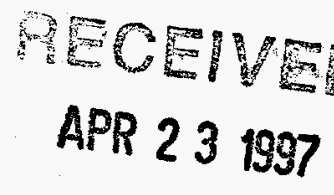

The Tokamak Fusion Test Reactor has operated since November of 1993 with a deuteriumtritium fuel mixture for selected discharges. The majority of the tritium has been introduced as energetic neutral atoms of up to $120 \mathrm{keV}$ injected by the neutral beam systems, with some of the twelve ion sources run on pure tritium and some on deuterium to optimize the fuel mixture in the core plasma. A maximum beam power of 39.6 megawatts has been injected, and deuterium-tritium fusion power production has reached 10.7 megawatts, achieving central fusion power densities comparable to or greater than those expected for the International Thermonuclear Reactor, and allowing the first studies of fusion-produced alpha particle behaviour in reactor grade plasmas. Energy confinement in deuterium-tritium plasmas is better than in similar deuterium plasmas for most plasma regimes. Innovative techniques to manipulate the plasma current and pressure profiles are permitting studies of enhanced confinement regimes.

\section{INTRODUCTION}

The largest tokamak in the U. S. magnetic confinement fusion research program is the Tokamak Fusion Test Reactor (TFTR). By far the most significant source of power to heat the confined plasma is a neutral beam injection system, which has been previously described (1). During the first nine years of this system's operation, it produced deuterium neutral beams at powers which eventually reached $33 \mathrm{MW}$, and on a few occasions lower powers with ordinary hydrogen (protium).

However, the goal of 45 years of world-wide fusion research, performed until recently in plasmas of protium or deuterium, has been the deuterium-tritium (D-T) burning reactor, sustained by heating from fusion-produced alpha particles. Operation in mixed D-T plasmas began with two limited objective plasma discharges in the Joint European Torus in 1991 (2), which produced a peak fusion power of 1.7 MW. TFTR began an extensive campaign with tritium in November of 1993 which has continued through the present.

TFTR has extended the fusion power performance to $10.7 \mathrm{MW}$, with up to 6.5 megajoules of fusion energy released in a pulse. At these levels, the total plasma heating arising from the alpha particles (which carry only a quarter of the fusion energy, with the remainder bourne by neutrons out of the plasma) is still much smaller than the total neutral beam power. However, the heating by the fusion alpha particles is concentrated in the core of these plasmas, where the fusion power density has reached levels as high as $2.8 \mathrm{MW} / \mathrm{m}^{3}$. This alpha particle power density, is comparable to that expected for the core of the International Tokamak Experimental Reactor. Thus, TFTR is able to study plasmas under fusion conditions which are quite similar to those that will one day prevail in the core of a fusion reactor.

\section{OPERATING EXPERIENCE IN D-T PLASMAS}

Since the tritium phase of TFTR began, over 780 D-T discharges have been studied, with the aid of more than 2,500 ion source shots with tritium. These D-T tokamak discharges were interspersed among more than $17,000 \mathrm{D}$ tokamak discharges, heated by tens of thousands of deuterium ion source shots. The D discharges were performed for purposes of comparison to similar D-T shots, for optimization of plasma parameters, and to condition the interior of the tokamak vessel. This conditioning has been found necessary to reduce the near-surface hydrogen isotopes available for recycling into the plasma. Most of the plasma regimes which have been found on TFTR to demonstrate enhanced confinement, stability, or fusion performance require low edge recycling.

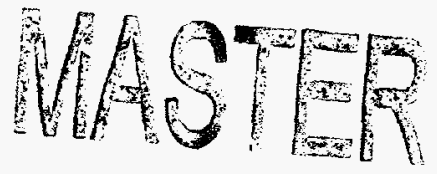




\section{Tritium Handling}

Modifications and manipulations of the neutral beams and the tritium handling system have become routine. Several hundred "line breaks" have been performed, during which tritium-containing volumes such as neutral beam ion sources or gas delivery systems have been decontaminated and opened for repair or maintenance activities.

About 89.8 grams of tritium have been processed by TFTR so far. Although some of this was puffed directly into the tokamak vacuum vessel, the great majority was introduced just downstream of the ion sources as a feedstock for producing ions and then neutralizing them. Only a few percent of this tritium enters the tokamak vessel, with the rest condensing on the liquid helium cryopanels of the four neutral beamlines. These panels are warmed up every few days, allowing the accumulated tritium and deuterium (of which there is much more) to be pumped away to the tritium handling system. At present this system oxidizes the mixed hydrogen isotopes to water, which is adsorbed onto molecular sieve beds, which are then shipped away for reprocessing.

This offsite shipment of the tritium is dictated by the fact that the TFTR tritium handling system is restricted to $50 \mathrm{kCi}$ of inprocess tritium. A cryodistillation system (3) is being commissioned which can repurify the tritium on-site, thus reducing the number of shipments required.

\section{Tritium Retention and Removal}

Careful accounting of tritium usage and recovery indicates that roughly $2 \%$ of the total throughput is held up in the torus and associated piping, representing a long term retention fraction of about $50 \%$ of the amount actually introduced to the torus as neutral beam particles and cold gas. Most of the tritium that lingers in the vacuum vessel is retained on the graphite limiter and co-deposited layers of carbon, lithium and hydrogenic isotopes on the walls.

The amount of tritium allowed in the TFTR vacuum vessel at any given time is restricted to less than $20 \mathrm{kCi}$ to limit the magnitude of a release to the environment in the event of a major vacuum leak and the simultaneous failure of tritium containment systems. If the tritium inventory caught in the vacuum vessel begins to approach this regulatory limit, then it is necessary to carry out cleanup operations. Retained tritium has been sucessfully removed from the TFTR vessel through glow discharge cleaning with deuterium, followed by glow discharge cleaning with a mixture of helium and oxygen, and then a moist air purge of the vessel (4). This reduced the inventory of long-term retained tritium from 16.4 $\mathrm{kCi}$ to about $8.1 \mathrm{kCi}$. With additional time devoted to glow discharge cleaning, the inventory could have been further reduced. The initial rate of tritium removal by deuterium glow discharge cleaning was $170 \mathrm{Ci} /$ hour, corresponding to the amount of tritium injected into the tokamak plasma by $\mathbf{4 6}$ megawatt seconds of tritium neutral beams.

An experiment was carried out to determine what the amount of releasable tritium would be in the event of an up-to-air accident with complete breach of confinement. In a planned moist air purge only about $10 \%$ of the in-vessel retained tritium was released from the surfaces where it was caught. This tritium was processed by the cleanup system (4). It appears that the great majority of the long-term retained tritium is tenaciously held, and is not readily releasable by an accidental exposure to moist air.

\section{Maintenance in the D-T Environment}

Operation with deuterium-tritium plasmas produces high fluxes of $14 \mathrm{MeV}$ neutrons, and the deuterium-deuterium reactions in these plasmas and in the far more numerous deuterium-only discharges produce $3.5 \mathrm{Mev}$ neutrons. TFTR, being an experimental device, was not built of the low-activation materials which would be used for a fusion power plant, and it does not have any remote handling capability. Nonetheless, machine activation has not prevented critical repair and maintenance activities.

One week after high power D-T operations, the radiation level near the tokamak vacuum vessel is in the range of $150 \mathrm{mrem} / \mathrm{hr}$. However, careful planning and use of local shielding as required has permitted repairs of such components as vacuum vessel leaks, toroidal field coil coolant leaks, and diagnostic actuators, all of which involved activities near the vacuum vessel. Moreover, the activation has not prevented the continuation of diagnostic calibrations. Farther away from the tokamak itself, most 
maintenance operations on the neutral beam systems have been little affected by the much lower activation levels present at distances of 10 14 meters from the TFTR vessel. Since the activation of relevance for maintenance is dominated by components with a half life of a few years, the activation accompanying continued operation at current neutron production rates will grow by only $20-30 \%$ per year, permitting continued access.

Maintenance on the neutral beams has been more significantly impacted by the presence of tritium adsorbed on internal surfaces. Before a component can be safely removed for repairs, it must be filled with moist air. Some of the adsorbed tritium on the interior surfaces of, for instance, an ion source, exchanges with hydrogen atoms in the water molecules, which can then be pumped away when the component is again evacuated. This pump and purge process is repeated several times, until the quantity of tritium being removed becomes sufficiently small to indicate that there is little adsorbed tritium left. The quantity of tritium removed from an ion source is usually about a curie. The purge batches with high tritium contamination are sent to the tritium cleanup system, while lowlevel batches can be vented to the site stack.

These pump and purge cleansing operations, along with monitoring and special precautions such as the use of portable elephant trunks to control air flow in the vicinity of maintenance activities, lengthen the time required to carry out any activity involving a "line break" of the tritium envelope. A reasonable rule of thumb for the sorts of procedures, such as ion source removal and replacement, which have become routine, is that the time required is about two to three times what was required before tritium was introduced. Nonetheless, this additional time has not proven to be an unreasonable burden, and many line breaks have been performed, principally to exchange ion sources or to make repairs to the neutral beam gas feed system.

\section{System Capability during D-T Operations}

Contrary to what might have been anticipated for the first ever attempt to use tritium for an extended period of time in a magnetic confinement fusion device, the tokamak and neutral beam capability have been enhanced during the D-T phase. The maximum toroidal field of the tokamak was increased from 5.2 to 6.0 Tesla at a major radius of $2.48 \mathrm{~m}$. This was accomplished by changing the configuration of the power supplies, increasing the maximum power extractable from the motor generator sets from 950 MVA to 1200 MVA, and undertaking tests to verify that the toroidal field coils could be operated beyond their nominal design point.

In addition, the maximum neutral beam power injected into the plasma was increased to $39.5 \mathrm{MW}$. This compares to a design rating of 33 MW and a previously obtained maximum of $\mathbf{3 4}$ $M W$ in deuterium operation. This increase arose partly from the fact that the higher neutralization efficiency of tritium relative to deuterium has more than made up for the lower optimum perveance, and thus lower extracted ion beam current, of tritium compared to deuterium. In addition, for the highest power D-T experiments the average operating voltage of the ion sources has been brought to higher levels than previously. For the highest power shots, the average tritium accelerator voltage was about $112 \mathrm{kV}$, and the average deuterium accelerator voltage about 107 $\mathrm{kV}$. This increase in capability is particularly striking in light of the fact that financial constraints have resulted in strategic parts, such as high voltage switch tubes, being used under conditions when they would once have been repaired, and being repaired at an age when they might once have been replaced.

- None of the failures which have occured have been related to tritium's radioactivity. They have instead been normal problems, such as water leaks in Langmuir probes in ion sources. There have been numerous problems with through-put leaks on the tritium gas valve system, but these problems did not arise due to any degradation caused by the tritium beta decays. Rather, they arose from the initial choice of valve seat material, and from the fact that this was the one part of the neutral beam system which had not undergone years of operations in the pre-tritium phase of TFTR.

\section{Tokamak Results}

The experimental program has concentrated on tokamak operating regimes which produce substantial fusion power (several MW), and thus can be used to explore alpha particle and D-T related issues under reactorrelevant conditions. 
Strachan and co-workers (5) demonstrated that aggressively conditioning the tokamak walls with helium discharges to reduce the recycling of deuterium and carbon from the limiters could lead to plasmas with improved energy confinement. These discharges, which came to be called "supershots," are characterized by centrally peaked density profiles and ion temperatures substantially higher than the electrons. Recently, lithium pellet injection has been used to coat the walls to further reduce hydrogenic ion and carbon influx, thereby extending the accessible operation range. With lithium conditioning, the plasma current in supershots has been increased to $2.7 \mathrm{MA}$ and the energy confinement time to about $330 \mathrm{msec}$. The fusion triple product of the energy confinement time, the central hydrogenic ion density, and the central ion temperature has reached a new record of $8.8 \times 10^{20} \mathrm{~m}^{-3} \mathrm{sec} \mathrm{keV}(6)$.

The increase in the fusion triple product was possible in part because of the finding that the energy confinement in D-T supershot plamas is significantly better than in similar discharges with deuterium only. For matched sets of discharges with all deuterium or all tritium neutral beam injection, the plasma stored energy and global energy confinement time are typically 25\% higher for the tritium beam cases. Most of this increase $(65-80 \%)$ arises from an increase in the stored energy of the thermal ions and electrons comprising the plasma. The remainder is due to the longer slowing down time of tritium beam ions relative to deuterium ones in the plasma. The strongest effect appears in the total effective ion thermal diffusivity (including convection), which drops by a factor of two in the central plasma (out to 0.7 of the minor radius). The momentum diffusivity and the electron particle diffusivity drop by a similar amount in this region, while the change in the electron thermal diffusivity is smaller and less certain (7).

The enhanced confinement and higher ion temperatures of the the D-T supershot plasmas allowed the fusion power released to reach a maximum of $10.7 \mathrm{MW}(8)$, while the total fusion yield from a single tokamak pulse has risen to 6.5 MJ. The central fusion power densities achieved in the best TFTR supershots, $2.8 \mathrm{MW} / \mathrm{m}^{3}$, are comparable to or greater than those expected in the International Tokamak Experimental Reactor. The ratio of the overall fusion power released to the neutral beam power injected has reached 0.27 . In addition, the first evidence of electron heating by the fusion alpha particles has been observed, with modeling suggesting that about half of the electron temperature rise in some plasmas is due. to energy transfer from the alphas.

In the highest performance supershot plasmas, the energy confinement has been sufficiently good that the maximum stored energy, and thus also the maximum fusion power output, is limited by the magnetohydrodynamic stability of the discharge. To deal with this problem, two different operating regimes, one called the high internal inductance mode, and the other the reversed shear mode, are being studied. In both cases, this is being done in part by transiently modifying the radial profile of the current flowing through the plasma, which in turn modifies the rate of change of the pitch of the total magnetic field (the vector sum of the internally produced polsidal magnetic field and the externally produced toroidal magnetic field).

The discharge duration is less than the current relaxation time in present high performance plasmas. Thus, varying the time evolution of the plasma current transiently alters the current profile. This has been accomplished on TFTR through ramping of the plasma current either up or down, and time variation of the neutral beam heating power in some cases. One of-the modes obtained, the high internal inductance mode, has a centrally peaked current profile. It has released a maximum of $8.7 \mathrm{MW}$ of fusion power, and is presently limited by confinement rather than stability. The other regime, the reversed shear mode, is characterized by a hollow plasma current profile. Under some conditions, this mode undergoes a transition to a regime of extremely high confinement; this regime is being studied for further optimization of its fusion power production.

*Supported by USDOE DE-AC02-76-CH0-3073

\section{REFERENCES}

1. Grisham, L. R., et al, Nucl. Instrum > and Meth. B1 0/11 478-481 (1985).

2. JET Team, Nucl. Fus. 32, 187-203 (1992).

3. Busigin, A.,et al, Fus. Tech. 28, 1312-20 (1995).

4. Skninner, C., et al, sub. to J. Vac. Sci. Tech (1996).

5. Strachan, J. D., et al, Phys. Rev. Lett. 58, 100410087(1987).

6. Mansfield, D. K, et al, Phys. . Plas. 2, 4252-4256 (1996).

7. Scott, S. et al, Phys. Plas. 2, 2308-2311 (1995).

8. McGuire, K et al, Phys. Plas. 2, 2176-2184 (1995). 


\section{DISCLAIMIER}

Portions of this document may be illegible in electronic image products. Images are produced from the best available original document. 